\title{
Normalized Point Source Sensitivity for Off-Axis Optical Performance Evaluation of the Thirty Meter Telescope
}

\author{
Byoung-Joon Seo ${ }^{a, b}$, Carl Nissly ${ }^{a, b}$, Mitchell Troy ${ }^{a, b}$, and George Angeli ${ }^{c}$ \\ ${ }^{a}$ Jet Propulsion Laboratory, Pasadena, CA. 91109, USA \\ ${ }^{b}$ California Institute of Technology, CA. 91125, USA \\ ${ }^{c}$ Thirty Meter Telescope Observatory, CA. 91107, USA
}

\begin{abstract}
The Normalized Point Source Sensitivity (PSSN) has previously been defined and analyzed as an On-Axis seeing-limited telescope performance metric. In this paper, we expand the scope of the PSSN definition to include Off-Axis field of view (FoV) points and apply this generalized metric for performance evaluation of the Thirty Meter Telescope (TMT). We first propose various possible choices for the PSSN definition and select one as our baseline. We show that our baseline metric has useful properties including the multiplicative feature even when considering Off-Axis FoV points, which has proven to be useful for optimizing the telescope error budget. Various TMT optical errors are considered for the performance evaluation including segment alignment and phasing, segment surface figures, temperature, and gravity, whose On-Axis PSSN values have previously been published by our group.
\end{abstract}

Keywords: Point Source Sensitivity, Optical Performance Metric, Optical Modeling, MACOS, Thirty Meter Telescope

\section{INTRODUCTION}

During the last few years, the Normalized Point Source Sensitivity (PSSN) has been introduced, studied, and utilized as a seeing-limited telescope performance metric by the Thirty Meter Telescope (TMT) systems engineering group. ${ }^{1-4}$ It is known that this metric is directly related to the photometric error of background limited observations so that it accurately represents the efficiency loss of science due to increased telescope observing time. $^{4,5}$ The PSSN metric also properly accounts for the consequences of different spatial frequency distributions for various wavefront error sources, which makes it superior to traditional metrics such as the root mean square of the wavefront error (RMS WFE) for estimating seeing-limited errors. Furthermore, its multiplicative feature (the combination of contributions from multiple error sources can be well estimated by multiplication of individually evaluated values.) makes this metric useful in designing and budgeting errors for a large telescope such as TMT, since its design and modeling tasks are highly distributed to multiple optical designers, vendors, and manufacturers.

However, the PSSN introduced so far is limited to only the On-Axis field of view (FoV) point. In investigating the Off-Axis telescope performance, the PSSN defined in the previous studies can be misleading due to several issues related to Off-Axis telescope characteristics such as vignetting and design aberrations. In this paper, we expand the scope of the PSSN definition more generally to include Off-Axis FoV points. We first find several issues in defining the PSSN at these Off-Axis field points including a Point Spread Function (PSF) normalization issue and a Point Source Sensitivity (PSS) normalization issue. We understand that the various choices for PSSN definition can be possible depending on these issues. Finally, we select one as a baseline among those possible PSSN definitions for the Off-Axis performance evaluation metric. Our intention is that we specify our baseline PSSN metric clearly here so that we can avoid further confusion for the future work we will perform.

The remainder of this paper is organized as follows. In Section 2 we first review the On-Axis definition of the PSSN. Section 3 develops and defines the notation for a more general PSSN definition. Section 4 shows the field dependent behavior of the proposed PSSN definition using both an aberrated and unaberrated TMT. Finally, we define our baseline PSSN in Section 5 based on the studies made throughout the paper.

Further author information: (Send correspondence to Byoung-Joon Seo)

Byoung-Joon Seo: E-mail: Byoung-Joon.Seo@jpl.nasa.gov

Modeling, Systems Engineering, and Project Management for Astronomy IV,

edited by George Z. Angeli, Philippe Dierickx, Proc. of SPIE Vol. 7738, $77380 \mathrm{G}$

(c) 2010 SPIE · CCC code: 0277-786X/10/\$18 - doi: 10.1117/12.857722

Proc. of SPIE Vol. $773877380 \mathrm{G}-1$ 


\section{ON-AXIS DEFINITION}

Suppose that $P S F_{t+a}$ is the time-averaged PSF of the unaberrated telescope looking through the atmosphere and $P S F_{t+a+e}$ is the time-averaged PSF of an aberrated telescope looking through the atmosphere. The OnAxis PSSN is defined ${ }^{1,2,4}$ as a ratio of the square integral of $P S F_{t+a+e}$ to that of $P S F_{t+a}$, and is formulated by Eq. (1).

$$
P S S N=\frac{\int_{\infty}\left|P S F_{t+a+e}(\vec{\theta})\right|^{2} d \vec{\theta}}{\int_{\infty}\left|P S F_{t+a}(\vec{\theta})\right|^{2} d \vec{\theta}}
$$

where $\vec{\theta}$ is the two dimensional coordinates for the PSF typically in units of radians (or meter). The subscripts $t$, $a$, and $e$ in $P S F_{t+a}$ and $P S F_{t+a+e}$ represent that the PSF is modified due to the telescope aperture, atmosphere, and alignment and surface errors, respectively. We will use this notation throughout this paper.

Since the Optical Transfer Function (OTF) and the PSF are related through the Fourier transformation according to the Parseval's energy conservation theorem, the PSSN can also be expressed using OTFs.

$$
P S S N=\frac{\int_{\infty}\left|O T F_{t+a+e}(\vec{f})\right|^{2} d \vec{f}}{\int_{\infty}\left|O T F_{t+a}(\vec{f})\right|^{2} d \vec{f}},
$$

where $O T F_{t+a}$ and $O T F_{t+a+e}$ are the OTFs of $P S F_{t+a}$ and $P S F_{t+a+e}$, respectively. Note that the integral square of OTFs in Eq. (2) is same as the H2 norm square of the optical system, i.e., $H_{2}^{2}=\int_{\infty}|O T F(\vec{f})|^{2} d \vec{f}$. The H2 norm is one commonly used way of quantizing the "size" of a linear optical system such as an optical telescope. Therefore, the system point of view of the PSSN representation is a ratio of the H2 norm square (or system size) of the aberrated telescope to relative to that of the unaberrated telescope.

We refer to our other publication ${ }^{4}$ for the detailed discussion on the philosophical background of its definition.

\section{GENERALIZING PSSN DEFINITION}

\subsection{Possible definitions \& terminology}

In defining the PSSN metric in a more general way including Off-Axis performance, several different definitions can be considered depending on the following two issues.

One is a PSF normalization issue. The time-averaged PSF such as in Eq. (1) represents an actual measurable physical quantity. Assuming unit emitting power (in Watts) from the point source and the unit exposure time (in seconds), it is in units of $\left[\mathrm{J} / \mathrm{m}^{2}\right]$ (or the number of photons as a function of pixel grid if it is represented on a digitized detector such as CCD) and includes both the energy loss (throughput) effect and spatial distribution of energy effect. In order to distinguish these two effects, it is often convenient to decompose the PSF as below.

$$
\operatorname{PSF}(\vec{\theta})=E \cdot \operatorname{psf}(\vec{\theta})
$$

where $E$ is the total energy the system collects from the point source and the $p s f(\vec{\theta})$ is the normalized PSF, i.e. $\int_{\infty}|\operatorname{psf}(\vec{\theta})| d \vec{\theta}=1$. Then, the right hand side of Eq. (1) becomes as follows.

$$
\frac{\int_{\infty}\left|P S F_{t+a+e}(\vec{\theta})\right|^{2} d \vec{\theta}}{\int_{\infty}\left|P S F_{t+a}(\vec{\theta})\right|^{2} d \vec{\theta}}=|\underbrace{\left(\frac{E_{e}}{E_{o}}\right)}_{\text {Throughput }}|^{2} \cdot \underbrace{\left(\frac{\int_{\infty}\left|p s f_{t+a+e}(\vec{\theta})\right|^{2} d \vec{\theta}}{\int_{\infty}\left|p s f_{t+a}(\vec{\theta})\right|^{2} d \vec{\theta}}\right)}_{P S S N}
$$

We call the energy ratio $\left(E_{e} / E_{o}\right)$ throughput. In order to avoid any confusion, we want to be clear that we distinguish the energy contribution (throughput) from the PSSN definition, making our PSFs normalized to one. Therefore, the defined PSSN considers only the spatial distribution of the energy. The energy loss effect (degradation or energy throughput) can be considered separately. Note that the energy loss can be significant if we consider vignetted Off-Axis FoV points. Nonetheless, we only considers its diffraction effect even for the Off-Axis PSSN evaluation. 
The other issue is a PSS normalization issue. The Off-Axis image quality degrades from that of the On-Axis due to inherent telescope design aberration, which we assume that we do NOT correct for. ${ }^{*}$ If the Off-Axis PSSN is defined such that it is normalized to its unaberrated On-Axis PSF, the Off-Axis PSSN will degrade even for the unaberrated telescope. We refer to this PSSN definition as $P S S N_{N}$ represented below.

$$
P S S N_{N}=\frac{\int_{\infty}\left|p s f_{t+a+e, \phi}(\vec{\theta})\right|^{2} d \vec{\theta}}{\int_{\infty}\left|p s f_{t+a, 0}(\vec{\theta})\right|^{2} d \vec{\theta}}
$$

where $p s f_{t+a, 0}$ is the time-averaged normalized PSF of the unaberrated On-Axis telescope and $p s f_{t+a+e, \phi}$ is the time-averaged normalized PSF of an aberrated On-Axis or Off-Axis $(\phi)$ telescope with atmospheric exposure. Alternatively the PSSN can be normalized to the Off-Axis PSS value itself. We refer to this PSSN definition as $P S S N_{F}$ and represent it as shown below.

$$
\operatorname{PSSN}_{F}=\frac{\int_{\infty}\left|p s f_{t+a+e, \phi}(\vec{\theta})\right|^{2} d \vec{\theta}}{\int_{\infty}\left|p s f_{t+a, \phi}(\vec{\theta})\right|^{2} d \vec{\theta}}
$$

where $p s f_{t+a, \phi}$ is the time-averaged PSF of the unaberrated Off-Axis telescope at the $\phi$ field point looking through the atmosphere.

We also define $P S S N_{N, o}$ and $P S S N_{F, o}$ as $P S S N_{N}$ and $P S S N_{F}$ when the telescope is unaberrated. Note that $P S S N_{N, o}$ depends only on the telescope design, which therefore is independent of the telescope aberration.

\subsection{Properties of various PSSN definitions}

Below are some properties associated with PSSNs we have defined.

P1 $P S S N_{F, o}$ (for the unaberrated telescope) becomes unity.

$$
P S S N_{F, o}=1
$$

P2 $P S S N_{N}$ is product of $P S S N_{N, o}$ and $P S S N_{F}$.

$$
P S S N_{N}=P S S N_{N, o} \cdot P S S N_{F}
$$

P3 $P S S N_{F}$ also has the multiplicative feature for low frequency and weak aberrations like the On-Axis definition of PSSN, ${ }^{2,4}$ i.e., Suppose there are multiple errors on the telescope. Let $P S S N_{\text {combined }}$ and $P S S N_{\text {multiplied }}$ be the PSSN for all combined error and for multiplied values of PSSN from individual errors, respectively. Then, $P S S N_{\text {multiplied }}$ approximates $P S S N_{\text {combined }}$.

$$
P S S N_{\text {combined }} \approx P S S N_{\text {multiplied }}
$$

$P 1$ and $P 2$ can be derived directly from their definitions while $P 3$ can be obtained from our studies performed later in this paper.

\section{FIELD DEPENDENT CHARACTERISTICS IN TMT}

We have calculated various optical performance metrics including $P S S N_{N}$ and $P S S N_{F}$ for the Off-Axis FoV of TMT. Section 4.1 and Section 4.2 show our calculation results for unaberrated and aberrated telescope status, respectively.

In computing the performance metrics, we have used the Modeling and Analysis for Controlled Optical Systems (MACOS). ${ }^{6}$ MACOS is a software tool for optical systems analysis developed at the Jet Propulsion Laboratory. MACOS has been used on projects such as JWST, SIM, TPF, and HST for optical and integrated modeling purposes. The MACOS tool has capabilities, which range from modeling complex surface geometries, atmospheric effects, and detector simulations using both sequential and non-sequential ray tracing and diffraction calculation.

\footnotetext{
${ }^{*}$ We assume that the inherent telescope design aberration is not corrected at Off-Axis points because of the following reasons. (A) The field corrector is not a part of the telescope we are evaluating, but a part of an instrument. (B) The field corrector cannot be always perfect, thus, should be understood or evaluated separately. We have an additional study in Appendix B assuming an ideal field corrector.
} 

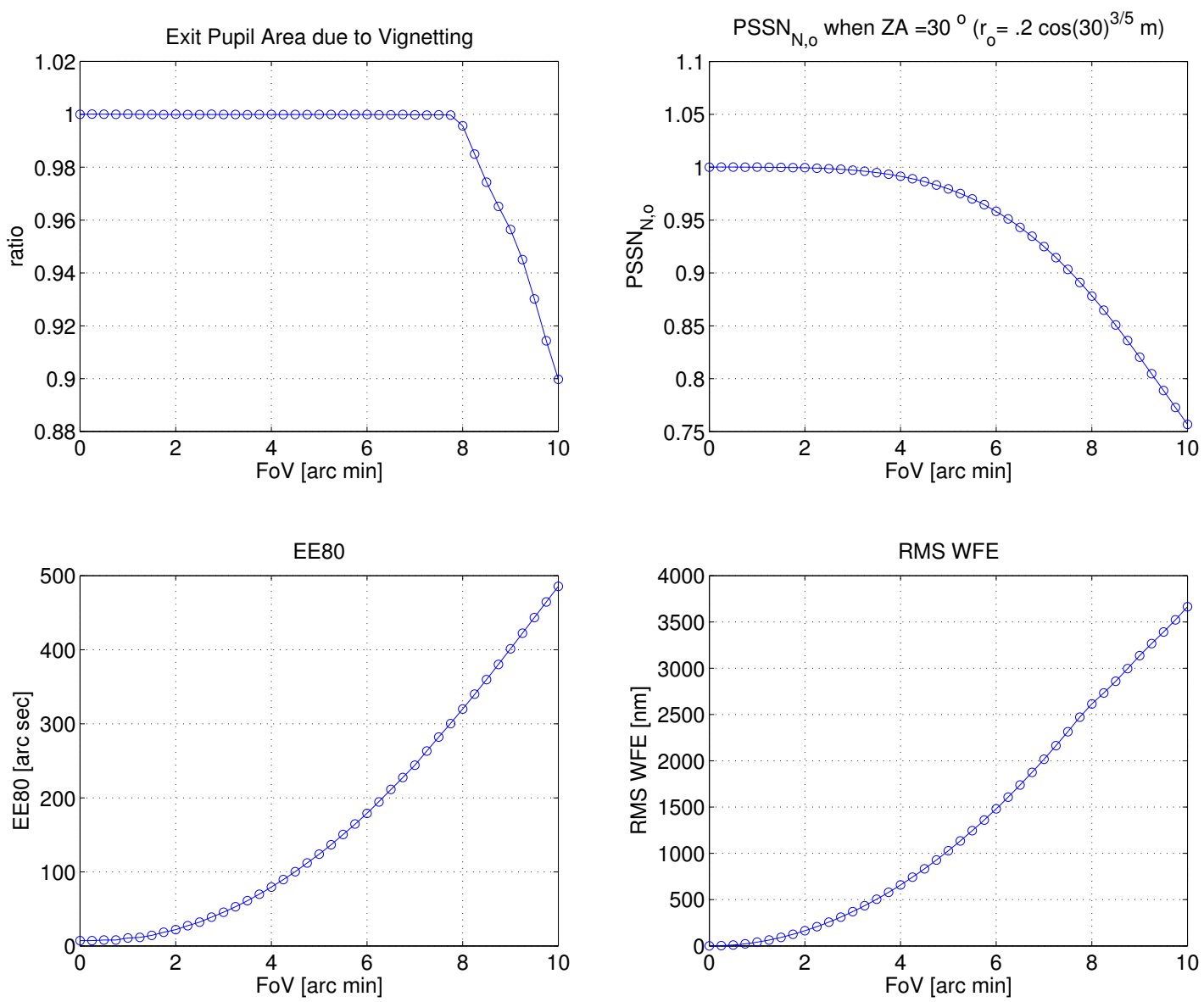

Fig. 1: Four subplots show the energy loss due to vignetting, unaberrated telescope $P S S N_{N, o}, 80 \%$ encircled energy diameter (EE80), and RMS WFE for this case as a function of radial distance of field-of-view (FoV) considered in Fig. 2. See Appendix A for the directional dependency of the vignetting.

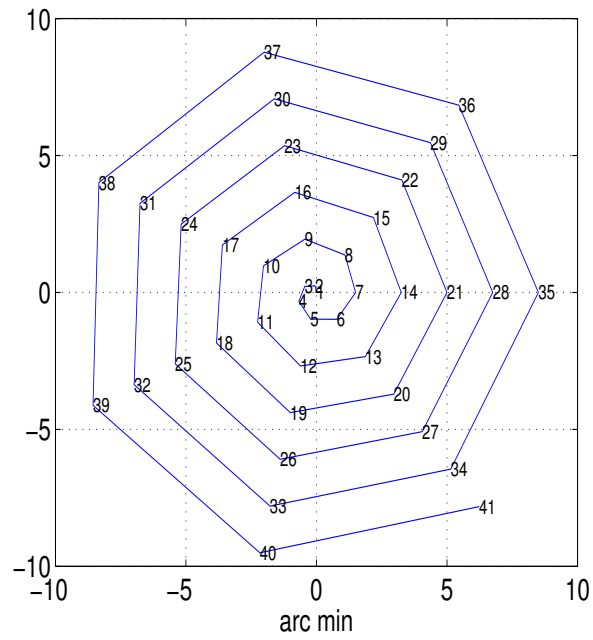

Fig. 2: The FoV points considered in this section spiral out from the On-Axis field point with increasing radius. 


\subsection{Unaberrated Ideal TMT Telescope}

The four subplots in Fig. 1 show the vignetting, $P S S N_{N, o}$, EE80, and RMS WFE as a function of radial distance of field-of-view (FoV) considered in Fig. 2. This vignetting is obtained from the exit-pupil area ratio to the OnAxis using unaberrated TMT telescope. It corresponds to throughput $\left(E_{e} / E_{o}\right)$ in Eq. (4) when the telescope is unaberrated. For the PSSN evaluation, we consider 30degree telescope pointing from the zenith with a value of the Fried parameter $r_{o}$ of $200 \mathrm{~mm}$ at the zenith. (The effective $r_{o}$ is then $200 \cdot \cos (30)^{3 / 5} \mathrm{~mm}$.)

We find that the impact of vignetting becomes apparent for half FoV (HFoV) values larger than 7.5arcmin. Note that the amount of vignetting depends on the direction of the Off-Axis angle due to the tilted Tertiary mirror. See Appendix A for the directional dependency of the vignetting.

\subsection{Aberrated TMT Telescope}

Fig. 3(a) shows numerically calculated $P S S N_{N}$ values due to the various M1 error sources in TMT. The M1 errors include passive support print-through (labeled as M1 passive support), correlated segment Piston/Tip/Tilt errors and phasing errors due to modeled performance of the Alignment and Phasing System (APS) (labeled

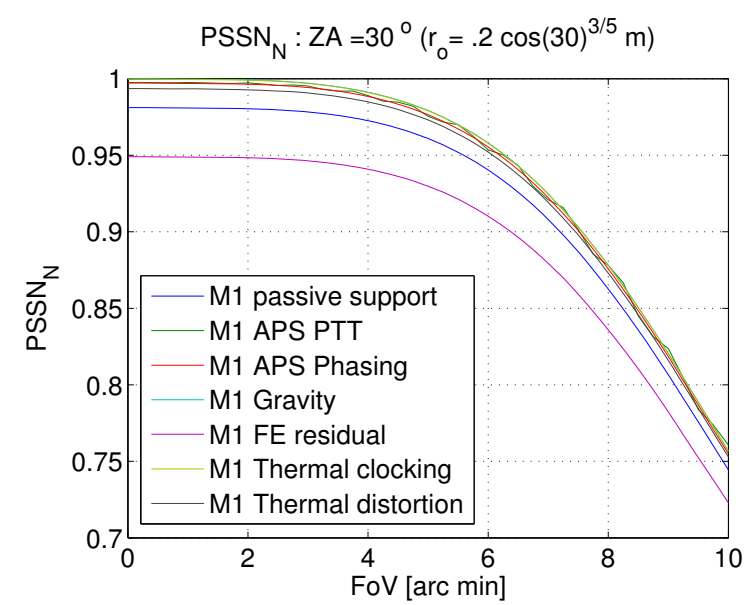

(a) $P S S N_{N}$

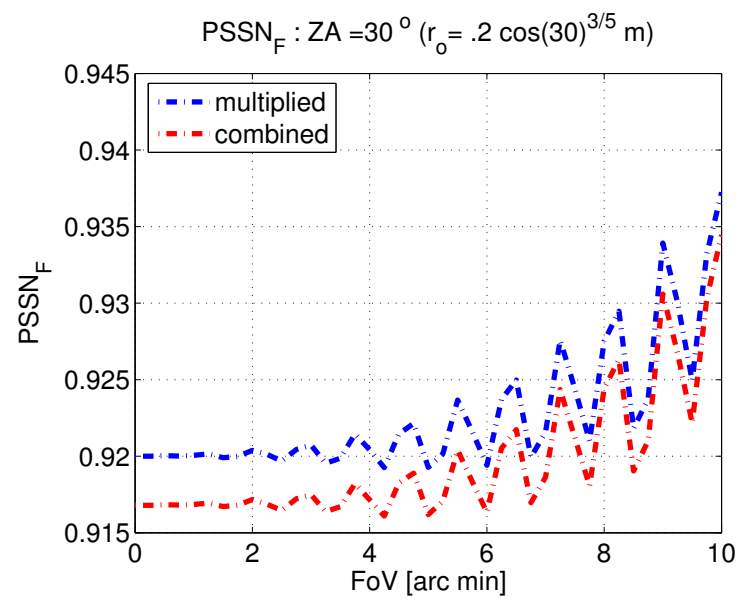

(c) Combined v.s. Multiplied

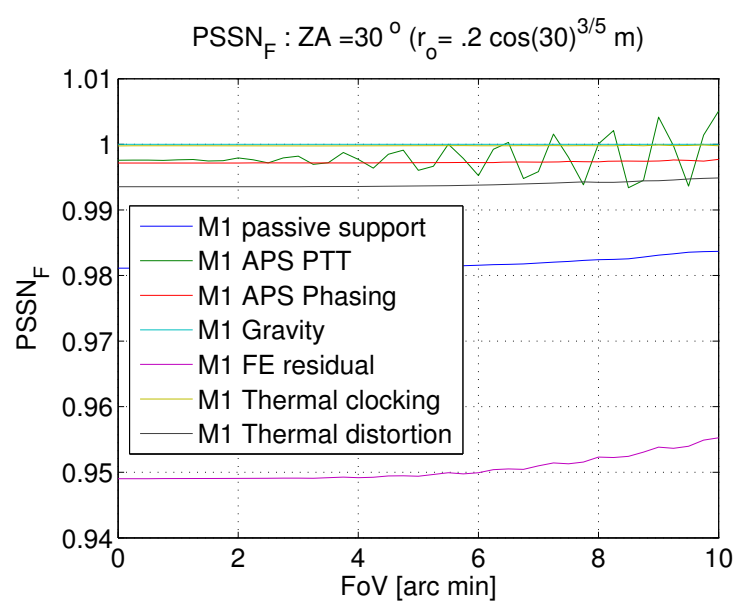

(b) $P S S N_{F}$

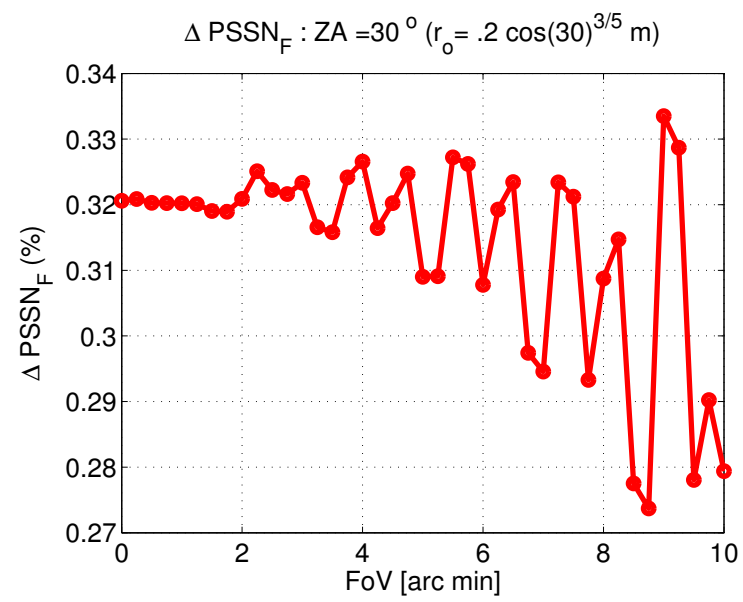

(d) Difference

Fig. 3: Numerically calculated $P S S N_{N}, P S S N_{F}$ values due to the various M1 error sources in TMT shown in (a) and (b). The combined and multiplied $P S S N_{F}$ and their difference are shown in (c) and (d) respectively demonstrating the multiplicative feature for the Off-Axis. See text for detail. 
as M1 APS PTT and M1 APS Phasing, respectively), gravity induced distortion and perturbation (labeled as M1 Gravity), segment figuring error residual after warping harness (labeled as M1 FE residual) and, segment thermal clocking and distortion (labeled as M1 Thermal clocking and M1 Thermal distortion, respectively). We have described these errors in detail in our previous studies. ${ }^{3}$ For the PSSN evaluation, we consider 30degree telescope pointing from the zenith with Fried parameter $r_{o}$ of $200 \mathrm{~mm}$ at the zenith. Using previously calculated $P S S N_{N, o}$ values in Fig. 1 and the property P2 in Section 3.2, we also obtain $P S S N_{F}$ values represented in Fig. 3(b).

In order to investigate the multiplicative feature of $P S S N_{F}$ for Off-Axis field points, we first combine all error sources and compute $P S S N_{F}$. We also multiply individually calculated $P S S N_{F}$ values, as shown in Fig. 3(b), to obtain multiplied values as a function of FoV. Fig. 3(c) and Fig. 3(d) show the combined and multiplied PSSNF values and their difference as a function of FoV respectively.

We have made the following observations in this study. First, as seen in Fig. 3(a), PSSN $N_{N}$ decreases as the FoV moves from the On-Axis field point due to inherent telescope design error (mostly astigmatism). Second, as seen in Fig. 3(b), $P S S N_{F}$ improves for larger radial field angles. This is also due to inherent telescope design errors, i.e. the same telescope surface errors impact the science metric less at larger Off-Axis field points than the same error does at a smaller Off-Axis point. Third, as seen in Fig. 3(b), PSSNF becomes larger than unity occasionally. This is because an induced error compensates the inherent telescope design error (mostly astigmatism) to improve the telescope performance. The effect is most evident for a low spatial frequency aberration such as M1 APS PTT error, where the fluctuation is because we consider the spiraled FoV positions ordered as shown in Fig. 2. This fluctuation will vary for different set of random M1 APS PTT errors. Note that we perform a Monte-Carlo study on these random errors in Appendix C. Fourth, as seen in Fig. 3(d), the multiplicative feature holds similarly for Off-Axis field points compared to the On-Axis case, which verifies the property P3 in Section 3.2.

\section{SUMMARY}

In summary, we define $P S S N_{F}$ as below.

$$
\operatorname{PSSN}_{F}=\frac{\int_{\infty}\left|p s f_{t+a+e, \phi}(\vec{\theta})\right|^{2} d \vec{\theta}}{\int_{\infty}\left|p s f_{t+a, \phi}(\vec{\theta})\right|^{2} d \vec{\theta}}
$$

where $\vec{\theta}$ is the two dimensional coordinates for the PSF typically in units of radians (or meter). The subscripts $t, a$, and $e$ in $p s f_{t+a, \phi}$ and $p s f_{t+a+e, \phi}$ represent telescope aperture, atmosphere, and alignment and surface errors at Off-Axis field angle $\phi$ for the normalized PSF (i.e. $\left.\int_{\infty}|(p s f \vec{\theta})| d \vec{\theta}=1\right)$. Therefore, $P S S N_{F}$ considers only the spatial distribution of the energy (excluding the energy loss) of an aberration and its Point Source Sensitivity (PSS) is normalized to the unaberrated telescope PSF at its own field point rather than to the On-Axis unaberrated PSF.

We make this decision for the following reasons. First, the Off-Axis normalization results in a multiplicative feature (See Section 4.2) while the On-Axis normalization does not. This multiplicative feature is highly useful for system performance estimations. ${ }^{3}$ Second, the PSSN values using the On-Axis normalization $\left(P S S N_{N}\right)$ can easily be obtained once we calculate the PSSN values using the Off-Axis normalization $\left(P S S N_{F}\right)$ by multiplying them with a separately calculated telescope design dependent PSSN value. (See Section 3.2 for detail) Finally, we have excluded the energy loss (throughput) effects from these PSSN values because the energy loss has a separate set of requirements, and their effects can always be included after $P S S N_{F}$ is obtained just like $P S S N_{N}$ can be calculated afterward using PSSN calculation for the inherent telescope design aberration $\left(P S S N_{N, o}\right)$.

\section{ACKNOWLEDGMENTS}

This research was carried out in part at the Jet Propulsion Laboratory, California Institute of Technology, and was sponsored by the California Institute of Technology and the National Aeronautics and Space Administration. The authors gratefully acknowledge the support of the TMT partner institutions. They are the Association of Canadian Universities for Research in Astronomy (ACURA), the California Institute of Technology and the 
University of California. This work was supported as well by the Gordon and Betty Moore Foundation, the Canada Foundation for Innovation, the Ontario Ministry of Research and Innovation, the National Research Council of Canada, the Natural Sciences and Engineering Research Council of Canada, the British Columbia Knowledge Development Fund, the Association of Universities for Research in Astronomy (AURA) and the U.S. National Science Foundation.

\section{APPENDIX A. BEAM VIGNETTING DIRECTIONAL DEPENDENCY}

Fig. 4 shows the vignetting as a function of radial distance of field-of-view (FoV). We have considered 4 different directions: $\pm \mathrm{X}_{\mathrm{M} 3 \mathrm{CRS}}$ (from On-Axis to $\pm \mathrm{X}$ direction) and $\pm \mathrm{Y}_{\mathrm{M} 3 \mathrm{CRS}}$ (from On-Axis to $\pm \mathrm{Y}$ direction). This vignetting is obtained from the exit-pupil area ratio to the On-Axis case using the unaberrated TMT telescope. This corresponds to $E_{e} / E_{o}$ in Eq. (4) when the telescope is unaberrated.

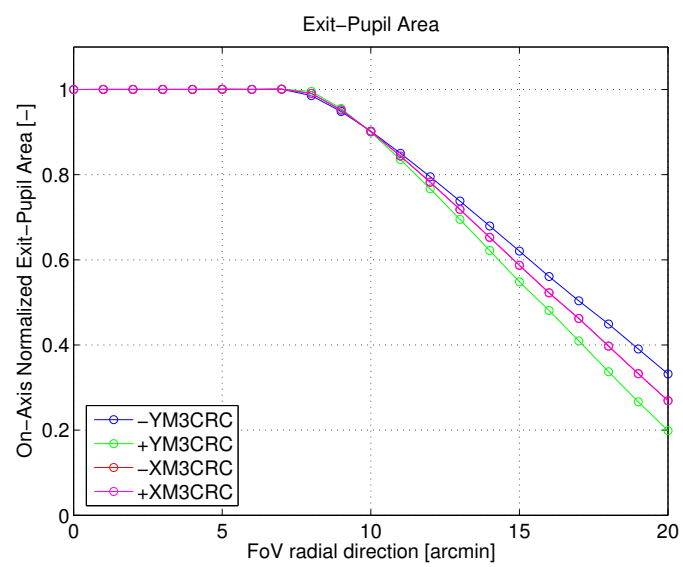

Fig. 4: The beam vignetting in $\mathrm{X}_{\mathrm{M} 3 \mathrm{CRS}}$ is symmetric, i.e., $-\mathrm{X}_{\mathrm{M} 3 \mathrm{CRS}}$ and $+\mathrm{X}_{\mathrm{M} 3 \mathrm{CRS}}$ vignetting are identical, while the beam vignetting in $\mathrm{Y}_{\mathrm{M} 3 \mathrm{CRS}}$ is asymmetric due to the M3 tilt causing different beam footprints.

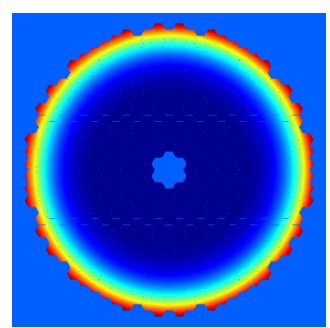

(a) On-Axis w/o Corrector

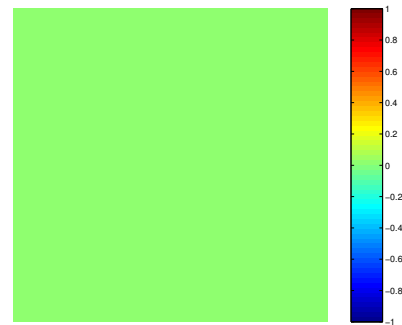

(b) On-Axis w/ Corrector

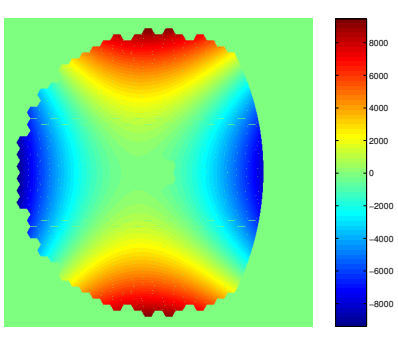

(c) Off-Axis w/o Corrector

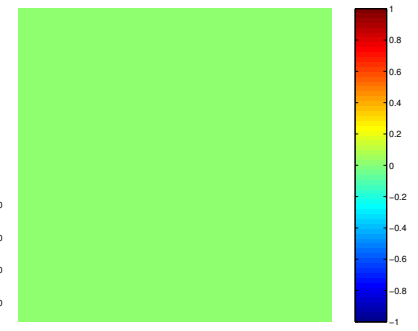

(d) Off-Axis w/ Corrector

Fig. 5: On-Axis or Off-Axis OPD with or without ideal Field Corrector for unaberrated telescope.

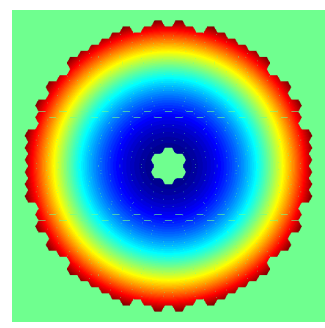

(a) On-Axis w/o Corrector
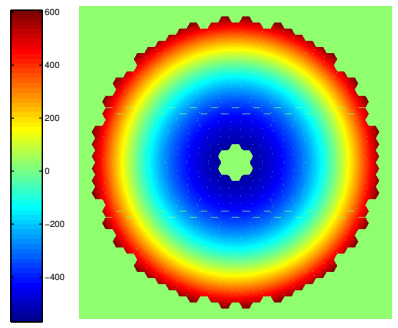

(b) On-Axis w/ Corrector
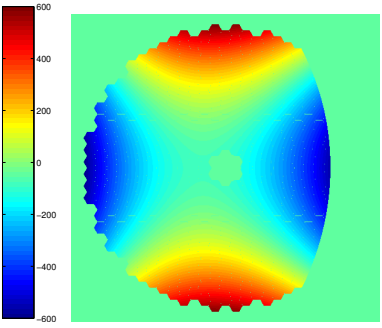

(c) Off-Axis w/o Corrector
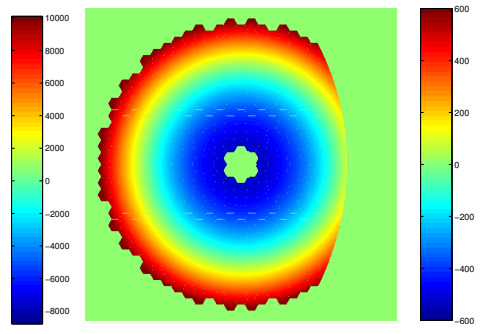

(d) Off-Axis w/ Corrector

Fig. 6: On-Axis or Off-Axis OPD with or without ideal Field Corrector for aberrated (M2 piston) telescope. 
We find that the vignetting impact becomes apparent for half FoV (HFoV) angles larger than 7.5arcmin regardless of the $\mathrm{FoV}$ direction. We also find that the vignetting in $\mathrm{X}_{\mathrm{M} 3 \mathrm{CRS}}$ is symmetric, i.e., - $\mathrm{X}_{\mathrm{M} 3 \mathrm{CRS}}$ and $+\mathrm{X}_{\mathrm{M} 3 \mathrm{CRS}}$ vignetting are identical, while the vignetting in $\mathrm{Y}_{\mathrm{M} 3 \mathrm{CRS}}$ is asymmetric due to the M3 tilt causing different beam footprints.

\section{APPENDIX B. PSSN WITH IDEAL FIELD CORRECTOR}

So far, we assume that the inherent telescope design aberration is not corrected for Off-Axis. This is due to the following reasons. (A) The field corrector is not a part of the telescope we are evaluating, but a part of an instrument. (B) The field corrector will not be always perfect, thus, should be understood or evaluated separately. In this section, we nonetheless consider ideal Field Corrector (FC) in defining the PSSN. By "ideal" FC, we mean that the inherent telescope design aberration is completed removed by the corrector. Fig. 5 shows the On-Axis and Off-Axis OPD with or without an ideal FC for the unaberrated TMT telescope. The similar plots are shown in Fig. 6 for the aberrated (M2 piston movement) TMT telescope.

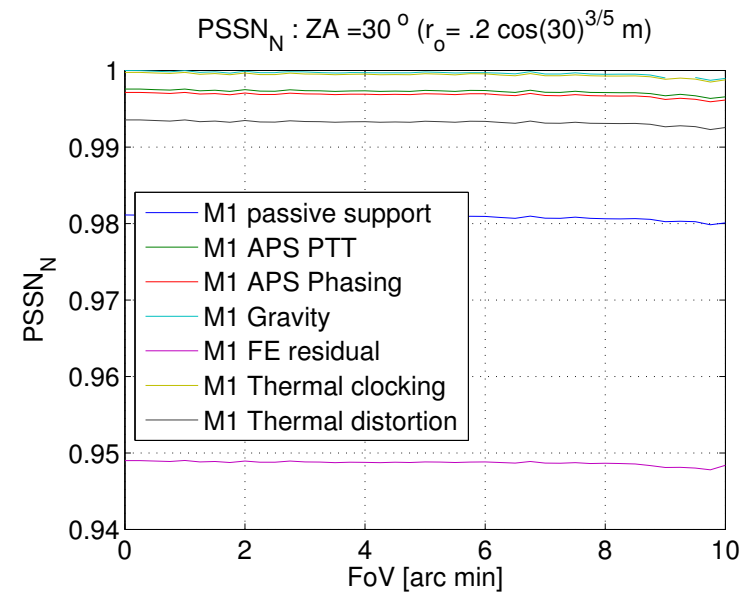

(a) $P S S N_{N}$

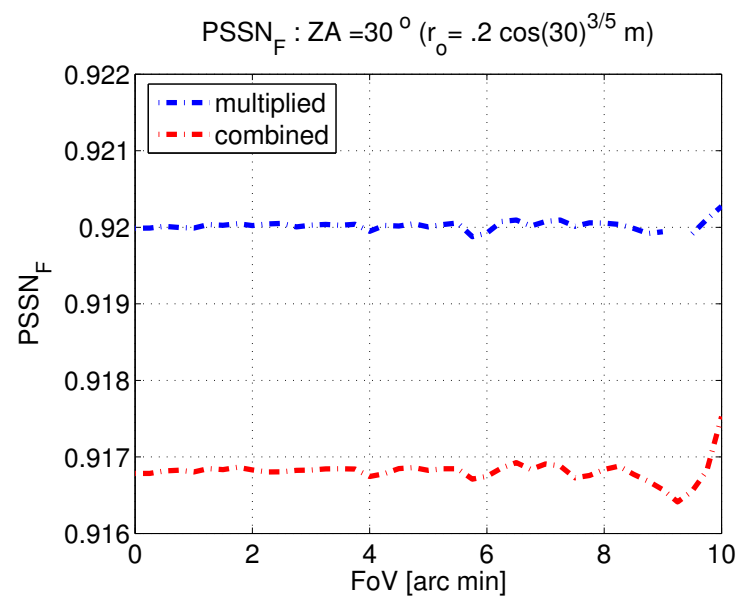

(c) Combined v.s. Multiplied

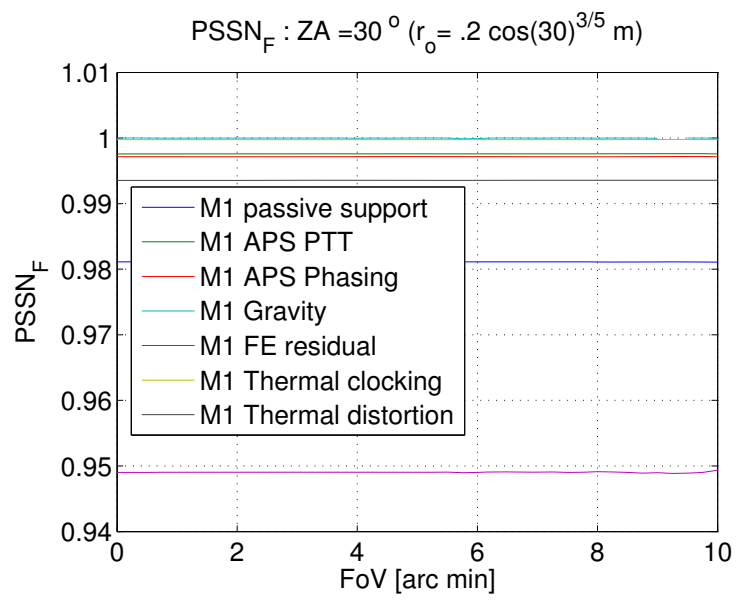

(b) $P S S N_{F}$

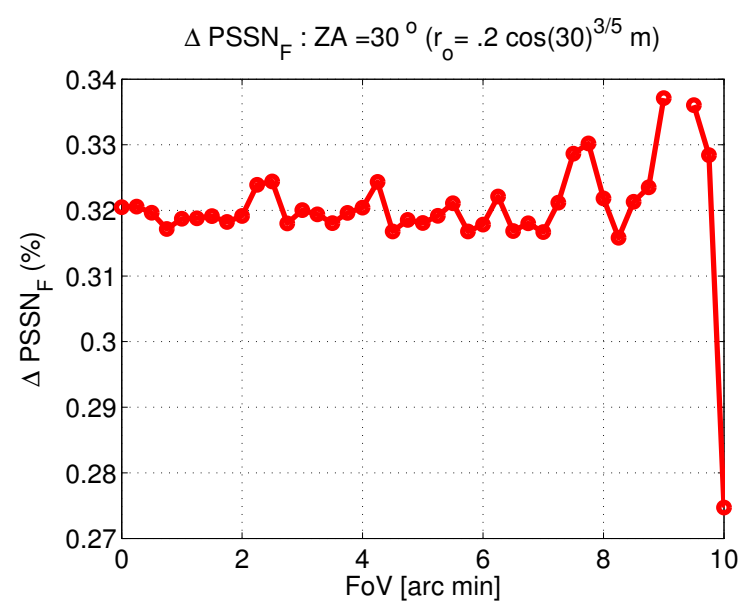

(d) Difference

Fig. 7: We consider the ideal FC to replot Fig. 3 :Numerically calculated $P S S N_{N}, P S S N_{F}$ values due to the various M1 error sources in TMT shown in (a) and (b). The combined and multiplied $P S S N_{F}$ and their difference are shown in (c) and (d) respectively demonstrating the multiplicative feature for the Off-Axis. 
Fig. 7 shows again computed PSSN values for various TMT error sources ${ }^{3}$ (comparable plots to Fig. 3) with considering the ideal FC. As seen in Fig. 7(a) and Fig. 7(b), installing ideal FC makes the PSSN values less dependent on FoV. Furthermore, we find that the multiplicative feature holds as well as seen in Fig. 7(d),

\section{APPENDIX C. PSSN WITH AVERAGING}

The PSSN value fluctuation in M1 APS PTT errors in Fig. 3(b) (also reproduced Fig. 8(a)) is because the error contains global astigmatism, which is compensated or intensified with the inherent telescope design aberration depending on the FoV. (We often call it "Mode canceling effect".)

The M1 APS PTT errors are random error, thus the PSSN value will vary if we consider other set of M1 APS PTT errors. We perform a Monte-Carol study on the random errors, i.e., many realizations (10 times in this study) to compute the averaged values in Fig. 8. Note that we consider other random errors as well such as M1 APS phasing error and M1 FE residual. Since these errors contain much higher spatial frequency aberration than astigmatism, the Monte-Carlo impact is not as significant as the M1 APS PTT error.

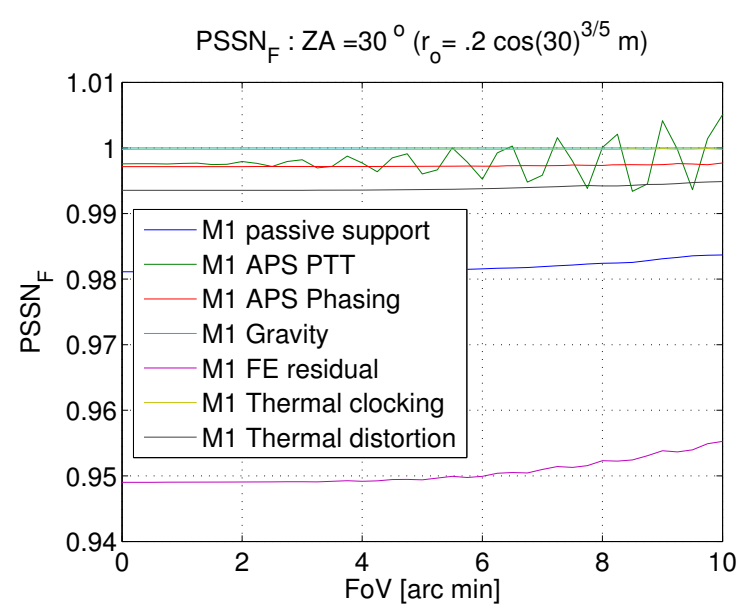

(a) $P S S N_{F}$ w/o averaging

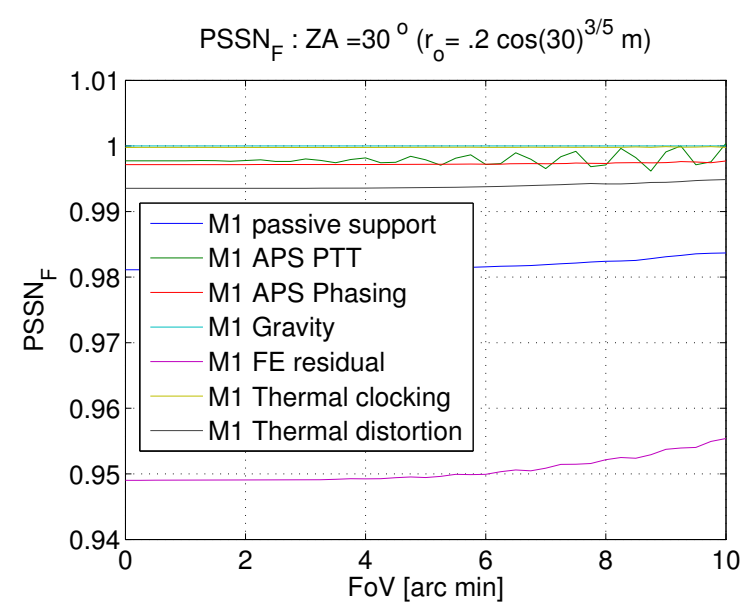

(b) $P S S N_{F} \mathrm{w} /$ averaging

Fig. 8: $P S S N_{F}$ with or without averaging.

\section{REFERENCES}

1. G. Z. Angeli, S. Roberts, and K. Vogiatzis, "Systems Engineering for the Preliminary Design of the Thirty Meter Telescope," Proc. SPIE 7017, p. 701704, June 2008.

2. B.-J. Seo, C. Nissly, G. Angeli, B. Ellerbroek, J. Nelson, N. Sigrist, and M. Troy, "Analysis of Normalized Point Source Sensitivity as a performance metric for the Thirty Meter Telescope," Proc. SPIE 7017, p. $70170 \mathrm{~T}$, June 2008.

3. C. Nissly, B. Seo, M. Troy, G. Angeli, J. Angione, I. Crossfield, B. Ellerbroek, L. Gilles, and N. Sigrist, "High-resolution optical modeling of the Thirty Meter Telescope for systematic performance trades," Proc. SPIE 7017, p. 70170U, June 2008.

4. B.-J. Seo, C. Nissly, G. Angeli, B. Ellerbroek, J. Nelson, N. Sigrist, and M. Troy, "Analysis of Normalized Point Source Sensitivity as performance metric for large telescopes," Applied Optics 48, pp. 5997-6007, 2009.

5. I. R. King, "Accuracy of measurement of star images on a pixel array," Publications of Astronomical Society of the Pacific 95, pp. 163-168, Feb 1982.

6. D. Redding et al., "MACOS manual (modeling and analysis for controlled optical systems)," NASA JPL D-9816, internal document 5, 1999 